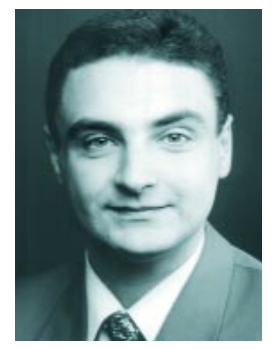

Alexei Korchounov

\title{
Therapie mit L-Dopa und retardiertem L-Dopa bei Patienten unter tiefer Hirnstimulation
}

\author{
Alexei Korchounov, Matthias Oechsner \\ Parkinson Klinik Bad Nauheim
}

psychoneuro 2004; 30 (8): 453-455

D ie wichtigsten Symptome des idiopathischen ParkinsonSyndroms sind Rigor, Bradykinese und Tremor. Die Patienten klagen oft über ein Zittern der Extremitäten, Gangstörungen und eine deutliche Einschränkung der Beweglichkeit, die mit einer Einschränkung der Fähigkeit zur Selbstversorgung verbunden ist. Die Häufigkeit der Erkrankung steigt mit dem Alter, allerdings können auch jüngere Menschen (bereits im 3. Lebensjahrzehnt) betroffen sein. Die Prävalenz in der Bevölkerung beträgt $0,2 \%$, sie steigt bei den über 65 jährigen auf $1 \%$ an und liegt bei den über 85 jährigen bei ca. $3 \%$. Männer erkranken etwas häufiger als Frauen (1).

Durch eine adäquate medikamentöse Behandlung kann in den ersten Jahren bei der Mehrheit der Patienten eine Linderung bzw. deutliche Verbesserung der Symptome erreicht werden. Im Verlauf entwickeln sich jedoch oft Komplikationen der medikamentösen Therapie, wie Fluktuationen der Beweglichkeit, Psychosen, orthostatische Hypotonie und gastrointestinale Störungen. Diese Zustände verschlechtern die Lebensqualität der Betroffenen und verursachen einen höheren pflegerischen Aufwand. In Spätstadien der Erkrankung benötigen die Patienten höhere Dosierungen der

Das Idiopathische Parkinson-Syndrom (IPS) ist eine progrediente Erkrankung. Durch eine adäquate medikamentöse Behandlung kann in den ersten Jahren bei der Mehrheit der Patienten eine Linderung bzw. deutliche Verbesserung ihrer Symptome erreicht werden. Im Verlauf entwickeln sich jedoch bei vielen Patienten die Komplikationen der medikamentösen Therapie: Fluktuationen der Beweglichkeit, Psychosen, orthostatische Hypotonie und gastrointestinale Störungen. Bei fehlender Möglichkeit der medikamentösen Einstellung stellt in schweren Fällen eine stereotaktische bilaterale Elektrodenimplantation in den Nucleus subthalamicus (STN) oder Nucleus ventralis intermedius (VIM) thalami eine wichtige therapeutische Option dar. Beide Verfahren werden mit einem Oberbegriff „Tiefe Hirnstimulation“(THS) bezeichnet. Dabei wird durch den regulierbaren Stromreiz die Aktivität bestimmter ZNS-Strukturen gezielt beeinflusst. Die tiefe Hirnstimulation verbessert oft die Symptome der Erkrankung und damit die Lebensqualität der Patienten. Bei vielen Erkrankten ist eine erhebliche Dosisreduktion der Parkinson-Medikamente möglich, wodurch die Häufigkeit der Nebenwirkungen sinkt. Abhängig von der Elektrodenposition und Stimulationsstärke können jedoch bei der tiefen Hirnstimulation Nebenwirkungen auftreten. Für Erreichen einer guten Beweglichkeit bei seltenen, unerwünschten Effekten hat sich eine Kombination aus der tiefen Hirnstimulation und medikamentöser Therapie als sinnvoll erwiesen. In vielen solchen Fällen trägt eine postoperative Behandlung mit Levodopa (z.B. Nacom oder Nacom retard) zur deutlichen Verbesserung des Zustandes der Patienten bei.

Substanzen, wodurch die Wahrscheinlichkeit der Nebenwirkungen steigt. Zusätzlich sind die Möglichkeiten der medikamentösen Therapie mit der Alterung der Erkrankten und dem Vorliegen von Begleiterkrankungen eingeschränkt. Bei fehlender Möglichkeit der medikamentösen Einstellung stellt in schweren Fällen eine stereotaktische neurochirurgische Operation eine wichtige therapeutische Option dar.

\section{Neurochirurgie}

Die erste bilaterale zervikale Wurzeldurchtrennung, um Rigor und Tremor zu beseitigen, wurde 1921 von Lerche beschrieben. 1937 wurde die erste Kortektomie bei einem Patienten mit Tremor durchgeführt. 1939 begann Meyers die ersten offenen Operationen an Basalganglien mit transventrikulärem Zugang. Diese Technik wurde in den 40-er Jahre wegen einer hohen postoperativen Mortalität aufgegeben. Aber diese Operationen zeigten, dass die Läsionen der Basalganglien die Parkinson-Symptome positiv beeinflussten. 1947 erfolgte die erste stereotaktische Pallidotomie. Im Laufe der 50-er Jahre wurden bevor- 


\section{Fallbeispiel}

Patient S., 61 Jahre alt. IPS seit zehn Jahren. Indikationen zur THS mit Elektrodenimplantation in den Nucleus subthalamicus bestanden in deutlichen ONOFF-Fluktuationen der Beweglichkeit und medikamenteninduzierter Psychose. Der Patient war rollstuhlpflichtig und benötigte Hilfe bei allen Aktivitäten des alltäglichen Lebens. Präoperativ wurde Herr S. mit einer Kombination von Apomorphin (Pumpe) und einem anderen Dopaminagonisten behandelt, zusätzlich erhielt er Clozapin. Der Termin der Operation musste wegen schwerer psychotischer Symptomatik mit fehlender Einwilligungsmöglichkeit dreimal verschoben werden. Postoperativ wurde zunächst versucht, parallel zur Erhöhung der Stimulationsparameter die Apomorphin-Pumpe abzusetzen und zur Verbesserung der Beweglichkeit die Dosis des Dopaminagonisten zu erhöhen, worauf der Patient erneut eine schwere Psychose entwickelte. Aus diesem Grund wurde der Patient auf eine Kombination aus Standard- und retardiertem Levodopa umgestellt. Unter dieser Therapie und gleichzeitiger Erhöhung der Stimulationsparameter bestanden bei Entlassung keine Fluktuationen der Beweglichkeit, der Patient war in der Lage, im Zimmer und auf der Stationsebene zu gehen, konnte allein essen und sich anziehen. Die psychotische Symptomatik sistierte, so dass die Dosis von Clozapin deutlich reduziert werden konnte.

zugt Operationen am ventrolateralen Thalamus und Nucleus subthalamicus durchgeführt. Diese Operationen gewannen in der Therapie des IPS zunehmend an Bedeutung: Weltweit wurden bis Ende der 60-er Jahre ca. 40000 Eingriffe durchgeführt. Nach Einführung von Levodopa und anderen Substanzen sank die Zahl der Stereotaxien deutlich ab. Zunehmende Erkenntnisse über die Pathogenese der Erkrankung, der Einführung der speziellen Elektroden für intrazerebrale Implantation und die zunehmende Zahl der Patienten mit ausgeprägten Nebenwirkungen der medikamentösen Therapie haben in den letzten Jahren zu einer Renaissance der stereotaktischen Techniken geführt (2).

Die stereotaktische bilaterale Elektrodenimplantation in den Nucleus subthalamicus oder in den Nucleus ventralis intermedius thalami stellen die modernen neurochirurgischen Methoden dar und werden als tiefe Hirnstimulation (THS) bezeichnet.
Nach den Leitlinien der Deutschen Gesellschaft für Neurologie besteht eine Indikation zur THS bei:

- Schwerem therapieresistenten IPS, insbesondere starkem Tremor

- Schweren therapieresistenten Fluktuationen der Beweglichkeit

- Ausgeprägten anderen Nebenwirkungen oder Unverträglichkeit der Parkinson-Medikamente, insbesondere substanzinduzierter Psychose.

\section{Auswahlkriterien für Patienten und präoperative Maßnahmen}

Nicht alle Patienten sind für die THS geeignet (3). Deswegen wird bei bestehenden Indikationen eine Selektion der Patienten durchgeführt. Ausgeschlossen werden die Patienten mit nicht-idiopathischen Krankheitsformen, Patienten mit Demenz sowie mit hirnorganischem Schaden oder ausgeprägter kortikaler Atrophie. Die Kandidaten für die Operation sollen auf die Gabe von Levodopa deutlich positiv reagieren (alle Symptome außer Tremor). Für die Auswahl der Patienten sind neben der allgemein-medizinischen und neurologischen Untersuchung folgende Maßnahmen notwendig:

- Neuropsychologische Tests zum Ausschluss einer Demenz (z.B. MMST > 25 Punkte)

- MRT zum Ausschluss einer anderen ZNS-Erkrankung als IPS, zur Darstellung der Zielregion und zur Berechnung der Trajektorien der Elektroden

- L-Dopa-Test 24 Stunden nach Absetzen aller Parkinson-Mittel (bei den Patienten, die mit Cabaseril behandelt werden, sollte dies 72 Stunden vor dem Test abgesetzt werden).

\section{Operationsablauf und -technik}

Die Operation erfolgt in zwei Schritten: Zunächst werden in Lokalanästhesie die Elektroden implantiert, danach erfolgt die subkutane Einbringung des Stimulators, meistens im infraklavikulären Bereich.

Die Elektroden besitzen vier Kontakte. Während der Einführung erfolgt eine kontinuierliche elektrophysiologische Registration. Da sich die ZNS-Strukturen in ihrem Aktivitätsmuster unterscheiden, kann die Position der Elektrode genau kontrolliert werden. Nachdem die Elektroden die Zielregion erreicht haben, erfolgt die intraoperative Testung der Elektrodenlage und der Stimulationseffekte. Dabei werden die klinischen Effekte bei der Stimulation jedes Kontaktes eingeschätzt. Je nach Effekt kann die Elektrodenposition korrigiert werden. Die Aktivierung mehrerer Kontakte erlaubt eine relativ breitflächige Stimulation der ZNS-Strukturen. Für jeden Kontakt kann man die Polarität, Stromstärke, Impulsfrequenz und Dauer des einzelnen Impulses einstellen. Nach Bestimmung der Kontakte mit dem besten klinischen Effekt wird der stereotaktische Teil der Operation abgeschlossen.

\section{Effekte und Nebenwirkungen der THS}

Bei einem erfolgreichen Eingriff und Einstellung der Stimulationsparameter ist die Verbesserung des $\mathrm{Zu}$ standes des Patienten durch die folgenden Effekte bedingt (4):

- Linderung der Kardinalsymptome des IPS (Bradykinese, Rigor, Tremor)

- Verlängerung der ON-Phasen ohne Hyperkinesen

- Verkürzung und geringere funktionelle Beeinträchtigung in OFF-Phasen

- Verbesserung der Lebensqualität

- Dosisreduktion der ParkinsonMedikamente mit Senkung der Häufigkeit der Nebenwirkungen.

Die THS kann jedoch zu Komplikationen führen. Manche sind durch Übertragung der elektrischen Stimulation an eng anatomisch benachbarte ZNS-Strukturen bedingt. Folgende Komplikationen können auftreten:

- Operative (Psychosyndrom, Infektion, Elektrodendislokation, Hauterosion)

- Technische (Elektrodenbruch)

- Stimulationsinduzierte Nebenwirkungen

- Gangunsicherheit

- Sprech- und Schluckstörungen

- Vermehrter Speichelfluss

- Blepharospasmus 
- Hemiparese

- Angst und Depression.

Je intensiver die Stimulation erfolgt, desto großer ist die Gefahr der Nebenwirkungen. Deswegen hat sich für Erreichen einer guten Beweglichkeit bei seltenen unerwünschten Effekten eine Kombination aus der tiefen Hirnstimulation und medikamentöser Therapie als sinnvoll erwiesen.

\section{- Postoperative Behandlung}

Unmittelbar nach der Operation ist eine Behandlung mit Levodopa aus zwei Gründen indiziert: Die Erhöhung der Stimulationsparameter kann oft nur langsam erfolgen. Die Gabe von Levodopa ermöglicht den Patienten den Erhalt der Beweglichkeit auf einem akzeptablen Niveau und beugt die Gefahr der Akinese vor. Außerdem ist bei der Monotherapie mit Levodopa das Risiko eines postoperativen Durchgangsyndroms im Vergleich zu den anderen dopaminergen Substanzen geringer.

Auch nach einer optimalen Einstellung der Stimulationsparameter trägt eine Behandlung mit Levodopa zur deutlichen Verbesserung des $\mathrm{Zu}$ standes der Patienten bei. Zwar bestehen verschiedene Möglichkeiten der medikamentösen Therapie nach der Operation, die Wahl der Substanzen ist jedoch von verschiedenen Faktoren abhängig (Alter, Psychose in der Vorgeschichte, andere Nebenwirkungen der Parkinsonmedikamente, Begleiterkrankungen). Die Therapie mit Levodopa ist insbesondere bei folgenden Fällen indiziert:

- Hohe Gefahr der Psychose

- Unverträglichkeit anderer Mittel

- Höheres Alter des Patienten

- Kombination mit anderen Parkinson-Medikamenten.

Durch die THS werden die kardinalen Symptome der Erkrankung positiv beeinflusst und die Patienten benötigen weniger Medikamente (5). Levodopa gilt auch heute noch als Goldstandard und eignet sich gut zur Ergänzung der Therapie, Spätkomplikationen treten nur selten auf, da die Dosis im Vergleich zum prä-operativen Zustand geringer gehalten werden kann. Auf der anderen Seite, reicht die Wirkung anderer Parkinson-Medikamente ohne Levodopa auch postoperativ oft nicht aus. Zwei Fallbeispiele aus unserer Klinik demonstrieren die wichtige Rolle von Levodopa bei Patienten nach der THS.

Therapy with Levodopa and sustained-release Levodopa of Patients under deep Brain Stimulation Idiopathic Parkinson Syndrome (IPS) is a progressive disease. In the majority of patients adequate drug treatment can achieve alleviation or definite improvement of the symptoms in the early years. In the later course, however, complications of drug treatment emerge in many patients: fluctuations of mobility, psychoses, orthostatic hypotension and gastrointestinal disorders. For patients who cease to respond to drug treatment there is a major therapeutic option in severe cases: stereotactic bilateral electrode implantation into the subthalamic nucleus (STN) or the ventral intermediate nucleus (VIM). Both these interventions are called „deep brain stimulation“. In this way a controllable electrical stimulus can be used to target and influence the activity of certain structures in the brain. Deep brain stimulation frequently improves the symptoms of the disease and hence the patient's quality of life. In many patients substantial reduction of the dosage of antiparkinsonian drugs is feasible, with a fall in the incidence of side effects. However, deep brain stimulation can have its own side effects, depending on the position of the electrodes and the strength of the stimulation. To achieve good mobility with minimal adverse effects a combination of deep brain stimulation and drug treatment has proved valuable. In many such cases postoperative treatment with Levodopa (e.g. Nacom or Nacom retard) contributes to a definite improvement in the patient's condition.

\section{Key Words}

Idiopathic Parkinson Syndrome (IPS) Deep brain stimulation - Levodopa Nacom - Nacom retard

\section{Fallbeispiel}

Patientin D., 65 Jahre alt. IPS seit zwölf Jahren. Indikationen zur THS mit Elektrodenimplantation in den Nucleus subthalamicus bestanden in deutlichen, therapieresistenten ON-OFF-Fluktuationen der Beweglichkeit. Insbesondere fiel ein starker OFF-Tremor auf. Die Patientin konnte in ON-Phasen mit einem Rolator gehen, in OFF-Phasen war sie rollstuhlpflichtig und benötigte Hilfe bei allen Aktivitäten des alltäglichen Lebens. Nach der Operation wurde parallel zur Einstellung der Stimulationsparameter ein Therapieversuch mit einer Kombination aus Dopaminagonist und Anticholinergikum durchgeführt. Die für eine gute Beweglichkeit notwendige $\mathrm{Er}$ höhung der Stimulationsparameter verursachte bei Frau D. ein Blepharospasmus, nach Aufdosierung des Dopaminagonisten trat eine orthostatische Hypotonie und gastrointestinale Störungen (Übelkeit, Erbrechen, Obstipation) auf. Deswegen musste sowohl die Stimulationstärke als auch die Dosis des Dopaminagonisten auf einem Niveau gehalten werden, auf dem keine Nebenwirkungen auftraten. Die Beweglichkeit der Patientin war dabei jedoch eingeschränkt und besserte sich erst nach zusätzlicher Gabe von Levodopa. Bei Entlassung konnte die Patientin zu jeder Tageszeit ohne Gehhilfe gehen und war in der Verrichtung von alltäglichen Aktivitäten selbstständig. Nebenwirkungen der THS oder medikamentösen Therapie bestanden nicht.

\section{Literatur}

1. Deuschl G, Krack P. Morbus Parkinson. In: Hopf GD, Diener HC, Reichmann H (Hrsg.). Neurologie in Klinik und Praxis, Handbuch der Neurologie in zwei Bänden. Stuttgart, Georg Thieme Verlag, 1999: 49-68

2. Ostertag CB, Lücking $\mathrm{CH}$, Mehdorn HM, Deuschl G. Stereotaktische Behandlung der Bewegungsstörungen. Der Nervenarzt 1997; 68: 477-484

3. Speelman JD, Bosch DA. Resurgence of functional neurosurgery for Parkinson's disease: a historical perspective. Mov Disord 1998; 13: 582-588

4. Toda H, Hamani C, Lozano A. Deep brain stimulation in the treatment of dyskinesia and dystonia. Neurosurg Focus 2004; 17 (1): E2

5. Volkmann J, Allert N, Voges J, Sturm V, Schnitzler A, Freund HJ. Long-term results of bilateral pallidal stimulation in Parkinson's disease. Ann Neurol 2004; 55: 871-875

Korrespondenzadresse:

Dr. A. Korchounov

Parkinson Klinik Bad Nauheim

Franz-Groedel-Str. 6

61231 Bad Nauheim

E-Mail: a.korchounov@parkinsonzentrum.de 